\title{
Occurrence and Potentially Zoonotic Genotypes of Enterocytozoon Bieneusi in Wild Rhesus Macaques (Macaca Mulatta) Living in Nanwan Monkey Island, Hainan, China: A Public Health Concern
}

\section{Wei Zhao}

Department of Parasitology, Wenzhou Medical University, Wenzhou, Zhejiang

\section{Huan-Huan Zhou}

Key Laboratory of Tropical Translational Medicine of Ministry of Education, Hainan Medical University, Haikou, 571199, China.

\section{Guang-Xu Ren}

Key Laboratory of Tropical Translational Medicine of Ministry of Education, Hainan Medical University, Haikou, 571199, China.

\section{Yu Qiang}

Key Laboratory of Tropical Translational Medicine of Ministry of Education, Hainan Medical University, Haikou, 571199, China.

\section{Hui-Cong Huang}

Department of Parasitology, Wenzhou Medical University, Wenzhou, Zhejiang

\section{Gang Lu ( $\nabla$ ganglu2018@163.com )}

Key Laboratory of Tropical Translational Medicine of Ministry of Education, Hainan Medical University, Haikou, 571199, China.

\section{Feng Tan}

Department of Parasitology, Wenzhou Medical University, Wenzhou, Zhejiang

\section{Research Article}

Keywords: Enterocytozoon bieneusi, M. mulatta, Hainan (China), Zoonotic

Posted Date: February 25th, 2021

DOl: https://doi.org/10.21203/rs.3.rs-235735/v1

License: (c) (1) This work is licensed under a Creative Commons Attribution 4.0 International License. Read Full License 


\section{Abstract \\ Background}

Enterocytozoon bieneusi, a microsporidian species, is a zoonotic pathogen found in both humans and animals. Here, we determined the prevalence, explored the different genotypes of E. bieneusi in wild rhesus macaques (Macaca mulatta) (Hainan Island of China), and assessed their zoonotic potential.

\section{Methods}

We collected 173 fecal specimens from wild M. mulatta living in Nanwan Monkey Island, Hainan, China. Subsequently, we identified and genotyped E. bieneusi using nested PCR analysis amplification of the internal transcribed spacer region (ITS) of the rRNA gene. Lastly, a neighbor-joining tree was built based on gene sequences from the ITS region of E. bieneusi.

\section{Results}

Of the 173 specimens from wild M. mulatta, 26 (15\%) were infected with E. bieneusi. We identified six genotypes of E. bieneusi, of which five were known: PigEBITS7 $(n=20), D(n=2)$, Type IV $(n=1)$, Peru6 $(n$ $=1)$, Henan-III $(n=1)$, and a novel genotype: HNM-IX $(n=1)$. From the phylogenetic analysis, the six genotypes identified here were all categorized into zoonotic group 1.

\section{Conclusion}

Based on the results taht the novel genotype falling under zoonotic group 1 and all the known genotypes found in humans, we conclude that the wild $M$. mulatta infected with E. bieneusi have a public health significance.

\section{Background}

Enterocytozoon bieneusi is a typical human-pathogenic microsporidia species that contaminates the enterocytes of the small intestine [1]. Although its general infection is described by chronic diarrhea, and malabsorption or no clinical signs in immunocompetent humans, it can result in enhanced increased fatality via chronic diarrhea in individuals with immunodeficiency, such as patients with Acquired Immune Deficiency Syndrome (AIDS) [2]. Different studies have shown that it appears in several animals (mammals, birds, and reptiles) and some environmental samples (water, soil, and food)[3]. Most human infections result from the zoonotic transmission of spores through either infected food or water[4].

Recent surveys incorporated genotype information of E. bieneusi and elaborated genotype distribution among human populations and animal hosts [3]. Different studies have observed substantial genetic 
diversity within this species through sequencing the single internal transcribed spacer (ITS) region of the rRNA gene [5]. To date, scientists have detected approximately 500 E. bieneusi ITS genotypes. Among these genotypes, 49 were found in both animals and humans [3]. All genotypes of E. bieneusi could be categorized into 13 clades [6]. Here, two large groups ( 1 and 2 ) that are composed of genotypes common in animals and humans are termed zoonotic. The remaining 11 groups ( 3 to 13) contain genotypes from specific hosts or wastewater [3]. Furthermore, E. bieneusi is generally detected in various wildlife, either in captive or free populations, with a wide variety of genotypes, both host-adapted and host-free [3, 7]. Thus, wildlife is also an ecological resource for several human/animal infections. Therefore, the primary focus of epidemiological surveys should involve the genotyping of $E$. bieneusi isolates from under-sampled animal hosts with human contact to expand our knowledge regarding human microsporidiosis epidemiology and support E. bieneusi population analysis.

Rhesus macaques (Macaca mulatta) are prevalent in Southeast Asia, where their geographic range overlaps extensively with that of humans [8]. We carried out this study in the Nanwan Monkey Island, Nanwan peninsula, Lingshui county, south coast of Hainan, China. Globally, this is the only island-type nature reserve for $M$. mulatta and is home to over 2,500 monkeys. This island has a primitive natural environment, which makes it a perfect place for monkeys. Since its establishment in 1965, it has become a popular tourist destination. However, there is a lack of published studies on E. bieneusi infection in the M. mulatta living in the Nanwan Monkey Island. Therefore, this study aimed at investigate the incidence and different genotypes of E. bieneusi present in the wild M. mulatta.

\section{Results}

\section{Infection rates of $E$. bieneusi in wild $M$. mulatta}

Twenty-six of 173 specimens from wild $M$. mulatta were positive for $E$. bieneusi since they amplified the ITS region of the rRNA gene, with an average infection rate of $15.0 \%$. Besides, the infection rate of $E$. bieneusi in monkeys less than one year of age $(19.4 \%$; $14 / 72)$ was higher than those animals older than one year $(11.9 \% ; 12 / 101)$. Meanwhile, out of all the positives, $14.2 \%(17 / 120)$ were females, and $17.0 \%$ $(9 / 53)$ were males. However, as illustrated in Table 1 , the infection rates difference were not statistically significant either by age or by gender. 
Table 1

Prevalences of E. bieneusiand distributions of genotypes in Macaca mulatta by age and gender.

\begin{tabular}{|c|c|c|c|c|}
\hline \multirow[t]{2}{*}{ Gender } & \multicolumn{4}{|c|}{ Positive no./Examined no.(\%)-genotype (n) } \\
\hline & $\begin{array}{l}\text { Less than one } \\
\text { years of age }\end{array}$ & Over one years of age & Total & $\begin{array}{l}\text { aStatistics } \\
\text { value }\end{array}$ \\
\hline Male & $\begin{array}{l}\text { 5/24(20.8)- } \\
\text { PiglTS7(5) }\end{array}$ & $\begin{array}{l}\text { 4/29(13.8)-PigITS7 (3); } \\
\text { Type IV (1) }\end{array}$ & $\begin{array}{l}\text { 9/53 (17.0)-PiglTS7 (8); } \\
\text { Type IV (1) }\end{array}$ & \multirow[t]{2}{*}{$\begin{array}{l}\chi^{2}=0.23 \\
P=0.63\end{array}$} \\
\hline Female & $\begin{array}{l}\text { 9/48(18.8)- } \\
\text { PigEbITS7(7); } \\
\text { D(2) }\end{array}$ & $\begin{array}{l}\text { 8/72(11.1)-PigITS7(5); } \\
\text { Peru } 6 \text { (1); HNM-IX (1); } \\
\text { Henan-III (1) }\end{array}$ & $\begin{array}{l}\text { 17/120 (14.2)-PigITS7(12); } \\
\text { D(2); Peru 6 (1); HNM-IX } \\
\text { (1); Henan-III (1) }\end{array}$ & \\
\hline Total & $\begin{array}{l}\text { 14/72(19.4)- } \\
\text { PigEbITS7(12); } \\
\text { D(2) }\end{array}$ & $\begin{array}{l}\text { 12/101(11.9)- } \\
\text { PiglTS7(8); Peru } 6(1) ; \\
\text { Type IV (1); HNM-IX (1); } \\
\text { Henan-III (1) }\end{array}$ & $\begin{array}{l}\text { 26/173 (15.0)-PigITS7(20); } \\
\text { D(2); Peru } 6 \text { (1); Type IV } \\
\text { (1); HNM-IX (1); Henan-III } \\
\text { (1) }\end{array}$ & \\
\hline
\end{tabular}

${ }^{\text {bStatistics }} \chi^{2}=1.88, P=0.17$

value

${ }^{a}$ Statistics value $=$ Male vs Female, ${ }^{b}$ Statistics value $=$ Less than one years of age vs Over one years of age, Bold = the values higher than that in the same group were shown in bold.

\section{Genotype distribution of $E$. bieneusi by gender and age}

Six genotypes were identified in the wild $M$. mulatta through sequencing and multiple sequence alignment. They included five known genotypes (PigEbITS7, Type IV, Peru 6, D, and Henan-III) and one novel genotype (HNM-IX). Among them, genotype PigEbITS7 was dominant, and was found in $76.9 \%$ $(20 / 26)$ of $E$. bieneusi isolates. All the remaining genotypes were at a lower frequency: $7.7 \%(2 / 26)$ for genotype D, and 3.8\% (1/26) each for genotypes Peru 6, Type IV, Henan-III, and HNM-IX. Subsequently, two genotypes (PigEbITS7 and D) were detected in the less than one-year-old animals, whereas five genotypes (PigEbITS7, Peru 6, Type IV, Henan-III, and HNM-IX) in the more than one-year-old animals. As demonstrated in Table 1, two genotypes (PigEbITS7 and Type IV) were predominant in males, whereas five (PigEbITS7, D, Peru 6, Henan-III, and HNM-IX) in females.

\section{Genetic relationships of ITS genotypes}

Novel genotype HNM-IX had one single nucleotide polymorphism (SNP), with genotype EbpC (AF076042) having it at nucleotide site 51 of the ITS region. As illustrated in Fig. 2, phylogenetic analysis revealed that all genotypes belonged to zoonotic group 1 . They were further sub-divided into different genotype sub-groups such as PigEbITS7 and D in subgroup1a; genotypes Peru 6 in subgroup 1b; genotype Type IV in subgroup 1c; and genotypes Henan-III and HNM-IX in subgroup $1 \mathrm{~d}$.

\section{Discussion}


Non-human primates (NHPs) are known to possess a high genetic relationship with humans, which makes them useful biomedical research models. NHPs might be vulnerable to human diseases, thereby acting as zoonotic reservoirs $[8,10]$. In 1997, the first case of transference of E. bieneusi infection was recorded between a human (afflicted with AIDS) and a rhesus monkey (afflicted with simian immunodeficiency virus) [11]. However, until 2011, there was a lack of studies on the occurrence of $E$. bieneusi in non-human primates at the genotype level [10]. Zhao et al., summarized 16 studies on the infection of $E$. bieneusi in NHPs from seven countries [8, 12]. Among them, seven studies included $M$. mulatta, and they were all from China, with a prevalence range from 4.2 to $31.1 \%$ [12-18]. For the first time, our study has detected E. bieneusi in wild M. mulatta from the Hainan Province of China, with a prevalence of $15.0 \%$. Generally, E. bieneusi has been found to be more prevalent in wild M. mulatta here than other wild NHPs, such as baboons from Kenya (12.3\%) [10], chimpanzees from Cameroon (4.5\%) and Kenya (2.6\%) [21], gorillas from the Central African Republic (4.0\%) [20], orangutans from Indonesia (2.0\%) [19], and five captive species of wild NHPs from the Qinling Mountains of China [17]. Our study showed that E. bieneusi was more prevalent in M. mulatta than farm monkeys from Henan $(6.8 \%)$, Guangxi (8.5\%), Sichuan (10.5\%), and zoo monkeys from Henan (12.5\%) in China [14-16, 22]. However, the prevalence of E. bieneusi in monkeys from Rwanda (18.0\%) and some cities in China, like Shanxi (18.2\%), Shanghai (26.7\%), Hebei (27.0\%), and Beijing (29.2\%) was higher than that observed in our study [14-16, 20, 22]. Additionally, there are two more studies that identified E. bieneusi infection in laboratory macaques in Beijing (25.6\%) and Guangxi (18.5\%), China, which were both higher compared with this study $[13,23]$. In fact, in Hainan, two studies were reported on captive long-tailed macaques infected with E. bieneusi, which were also more than that observed in our study $[8,24]$. Similar to humans and farm animals, age substantially increases the risk E. bieneusi infection in NHPs [8]. Here, we identified a elevated E. bieneusi infection rate in young M. mulatta compared with adults, which agreed with the results of captive long-tailed macaque and laboratory macaques from Hainan, China and North China, respectively $[8,14]$. In addition to age, the health of the hosts, the detection methods, sample size, the experimental design, animal practices, etc. could cause the increase in prevalence.

Among the five known genotypes in our study, the genotype PigEbITS7 was detected in $76.9 \%(20 / 26)$ of E. bieneusi isolates, which shows predominance in the investigated wild M. mulatta. This genotype was initially detected in pigs from the USA [7] and it has been confirmed to have a broad host range, even in humans [3]. In China, PigEBITS7 was detected in some patients, including AIDS and hospitalized children, and several animals such as rodents, NHPs, and urban wastewater [5, 8, 25-27].

Additionally, previous studies have reported the presence of four other genotypes (Type IV, Peru6, D, and Henan-III) in humans and animals around the world, of which genotypes D and Type IV are commonly found in E. bieneusi- induced microsporidiosis in humans [3, 28]. Both genotypes D and Type IV have been detected in infants, HIV-positive patients, and HIV-negative patients in China [25, 29-33]. Meanwhile, they have been found in NHPs, pigs, dogs, snakes, cats, hippopotamus, Pere David's deer, chinchillas, Siberian tiger, lions, Fischer's lovebird, red foxes, wastewater, and lake water [3]. 
Genotypes Peru 6 (syn. PtEbl, PtEbVII) (from Peru and Portugal) and Henan-III (from Malaysia and China) have been spread across limited geographical area as well as small number of $E$. bieneusi-infected human cases compared with genotypes D and Type I [34-36]. Meanwhile, genotype Peru 6 has been identified in sheep, goats, reindeers, and wastewater [37-40], whereas genotype Henan-III has been found in NHPs, pet snakes, pigs, and birds in China [41-44]. Therefore, the above shreds of evidence suggest the possible zoonotic transmission of these genotypes from the wild M. mulatta to humans.

In this study, the novel genotype HNM-IX was genetically closely related to the human-pathogenic genotype EbpC which was commonly found in humans from Iran, Czech Republic, Peru, China, Thailand, and Vietnam [29, 45-48]. It was also found in more than 15 animal species and in environmental samples $[3,49]$. From the phylogenetic analysis, the six genotypes identified here were all categorized into group 1. Group 1 had almost all human-pathogenic genotypes and possessed $94 \%$ of the known $E$. bieneusi ITS sequences [3]. Therefore, the genotypes in wild macaques investigated including the novel one could have a sizeable zoonotic possibility.

\section{Conclusions}

This study is the first report to detect E. bieneusi infection in wild M. mulatta from Hainan, China. Humanpathogenic genotypes PigEbITS7, D, Type IV, Peru6, and Henan-III in these animals support a zoonotic nature for $E$. bieneusi. Here, the results of the phylogenetic analysis of the novel genotype falls into group 1 , which suggests a zoonotic possibility. Thus, visitors, veterinary workers, and the management of the wild M. mulatta should be educated and informed to minimize the risk for transmission of $E$. bieneusi from those animals.

\section{Methods}

\section{Fecal sample collection}

We obtained 173 stool samples from M. mulatta in Nanwan Monkey Island, located on the Nanwan peninsula, Lingshui county, south coast of Hainan, in the southernmost province of China. It is geographically located at $109^{\circ} 48^{\prime}$ east longitude and $18^{\circ} 29^{\prime}$ north latitude (Fig. 1). In this study, we used sterile disposable latex gloves to collect fresh fecal samples, which were then placed in marked plastic cups and stored at $4^{\circ} \mathrm{C}$.

\section{DNA extraction}

We sieved the fecal specimens, concentrated the filtrates, washed them thrice with distilled water, and centrifuged (10 min, 1500g). Then, we used a QIAamp DNA Stool Mini Kit (Qiagen, Hilden, Germany) to extract genomic DNA from washed fecal specimens (180-200 mg) following manufacturer's guidelines. Finally, DNA was eluted in $200 \mu \mathrm{L}$ of $\mathrm{AE}$ buffer and stored at $-20^{\circ} \mathrm{C}$.

\section{PCR amplification}


We analyzed all DNA preparations for $E$. bieneusi using nested PCR amplification. This amplification contained a nucleotide fragment (389 bp) containing 3' end small subunit (SSU) (76 bp), ITS region (243 bp), and 5 ' region of the large subunit (LSU) (70 bp) from E. bieneusi rRNA gene. Primers and cycle parameters were designed by Buckholt et al. (2002) [9]. All PCR tests used Taq DNA polymerase as well as a negative control (no DNA). Finally, all PCR products were separated via $1.5 \%$ agarose gel electrophoresis, followed by ethidium bromide staining.

\section{Nucleotide Sequencing}

All appropriately sized PCR products were purified using the Big Dye Terminator v3.1 Cycle Sequencing Kit (Applied Biosystems, USA) on an ABI PRISM 3730 XL DNA Analyzer (Sinogeno- max Biotechnology Co. Ltd., Beijing, China), followed by direct sequencing using PCR primers. We performed bidirectional sequencing to verify sequence accuracy.

\section{Sequence analysis}

We determined E. bieneusi genotypes. Here, we aligned the nucleotide sequences with each other, and used the BLAST and Clustal X 1.83 to access the reference sequences. In particular, the first published names corresponded to the sequences who had a 100 percent resemblance to those from known genotypes. Otherwise, they were described as novel genotypes. Finally, the nomenclature was established by naming all genotypes according to the $243 \mathrm{bp}$ of the ITS gene region of E. bieneusi [5].

\section{Phylogenetic analysis}

Here, we studied the genetic association between the novel and known genotypes. Next, we used the Mega X software (http://www.megasoftware.net/) to compare the ITS region of all identified nucleotide sequences with those of the reference sequences. The neighbor-joining tree was built based on the evolutionary distances calculated using a Kimura 2-parameter model and bootstrap analysis of 1,000 replicates.

\section{Abbreviations}

AIDS: acquired immune deficiency syndrome; SNP: single nucleotide polymorphism; NHPs: non-human primates; BLAST: Basic Local Alignment Search Tool; ITS: internal transcribed spacer; SSU: small subunit LSU: large subunit

\section{Declarations}

\section{Acknowledgements}

Not applicable.

Ethics approval and consent to participate 
The Research Ethics Committee and the Animal Ethics Committee of Hainan Medical University approved the study protocol. All animal experiments complied with the guidelines provided by the Regulations for the Administration of Affairs Concerning Experimental Animals.

\section{Consent for publication}

Not applicable.

\section{Availability of data and materials}

All data generated or analysed during this study are included in this published article. The identified nucleotide sequence of the novel genotype was submitted to the GenBank database (accession\# MW551790).

\section{Competing interests}

The authors declare that they have no competing interests.

\section{Funding}

This work was supported partially by the Open Foundation of Key Laboratory of Tropical Translational Medicine of Ministry of Education, Hainan Medical University (2020TTM004); the National Natural Science Foundation of China (No.82060375) and Innovation Research Team Project of Hainan Natural Science Foundation (2018CXTD340). The sponsors played no roles in the study design, or in the collection, analysis, or interpretation of the data, in writing the report, or in the decision to submit the article for publication.

\section{Authors' contributions}

WZ, FT and GLFT and GL

\section{References}

1. Fadhilah A, Gabbar A, Bokhari AA. Microsporidium. In: StatPearls. Treasure Island (FL): StatPearls Publishing; September 4, 2020.

2. Li W, Xiao L. Ecological and public health significance of Enterocytozoon bieneusi. One Health. 2020;12: 100209.

3. Li W, Feng Y, Santin M. Host Specificity of Enterocytozoon bieneusi and Public Health Implications. Trends Parasitol. 2019;35:436-451.

4. Fayer R, Santin-Duran M. "Epidemiology of microsporidia in human infections," in Microsporidia: Pathogens of Opportunity, 1st Edn, eds L. M. Weiss and J. J. Becnel (Chichester: John Wiley \& Sons, Inc.), 2024;1-64. doi.10.1002/9781118395264\$4 
5. Santín M, Fayer R. Enterocytozoon bieneusi genotype nomenclature based on the internal transcribed spacer sequence: a consensus. J Eukaryot Microbiol. 2009;56:34 - 8.

6. Zhao W, Zhou H, Yang L, Ma T, Zhou J, Liu H, et al. Prevalence, genetic diversity and implications for public health of Enterocytozoon bieneusi in various rodents from Hainan Province, China. Parasit Vectors. 2020;13:438.

7. Leśniańska K, Perec-Matysiak A. Wildlife as an environmental reservoir of Enterocytozoon bieneusi (Microsporidia) - analyses of data based on molecular methods. Ann Parasitol. 2017;63:265-281.

8. Zhao W, Zhou H, Jin H, Sun L, Li P, Liu M, et al. Genotyping of Enterocytozoon bieneusi among captive long-tailed macaques (Macaca fascicularis) in Hainan Province: High genetic diversity and zoonotic potential. Acta Trop. 2020;201:105211.

9. Buckholt MA, Lee JH, Tzipori S. Prevalence of Enterocytozoon bieneusi in swine: an 18-month survey at a slaughterhouse in Massachusetts. Appl Environ Microbiol. 2002;68:2595-9.

10. Li W, Kiulia NM, Mwenda JM, Nyachieo A, Taylor MB, Zhang X, et al. Cyclospora papionis, Cryptosporidium hominis, and human-pathogenic Enterocytozoon bieneusi in captive baboons in Kenya. J Clin Microbiol. 2011;49:4326-9.

11. Tzipori S, Carville A, Widmer G, Kotler D, Mansfield K, Lackner A. Transmission and establishment of a persistent infection of Enterocytozoon bieneusi, derived from a human with AIDS, in simian immunodeficiency virus-infected rhesus monkeys. J Infect Dis. 1997;175:1016-20.

12. Yu M, Liu X, Xie M, Li D, Ni Q, Zhang M, et al. Epidemiological investigation and genotypes of Enterocytozoon bieneusi in 11 captive Rhesus macaque populations. Int $\mathrm{J}$ Parasitol Parasites Wildl. 2020;13:191-195.

13. Yang H, Lin Y, Li Y, Song M, Lu Y, Li W. Molecular characterization of Enterocytozoon bieneusi isolates in laboratory macaques in north China: zoonotic concerns. Parasitol Res. 2017;116:28772882.

14. Zhong Z, Li W, Deng L, Song Y, Wu K, Tian Y, et al. Multilocus genotyping of Enterocytozoon bieneusi derived from nonhuman primates in southwest China. PLoS One. 2017;12:e0176926.

15. Karim MR, Wang R, Dong H, Zhang L, Li J, Zhang S, et al. Genetic polymorphism and zoonotic potential of Enterocytozoon bieneusi from nonhuman primates in China. Appl Environ Microbiol. 2014;80:1893-8.

16. Karim MR, Wang R, Dong H, Zhang L, Li J, Zhang S, et al. Genetic polymorphism and zoonotic potential of Enterocytozoon bieneusi from nonhuman primates in China. Appl Environ Microbiol. 2014;80:1893-8.

17. Du SZ, Zhao GH, Shao JF, Fang YQ, Tian GR, Zhang LX, et al. Cryptosporidium spp., Giardia intestinalis, and Enterocytozoon bieneusi in captive non-human primates in Qinling Mountains. Korean J Parasitol. 2015;53:395-402.

18. Ye J, Xiao L, Ma J, Guo M, Liu L, Feng Y. Anthroponotic enteric parasites in monkeys in public park, China. Emerg Infect Dis. 2012;18:1640-3. 
19. Mynářová A, Foitová I, Kváč M, Květoňová D, Rost $M$, Morrogh-Bernard $\mathrm{H}$, et al. Prevalence of Cryptosporidium spp., Enterocytozoon bieneusi, Encephalitozoon spp. and Giardia intestinalis in wild, semi-wild and captive orangutans (Pongo abelii and Pongo pygmaeus) on Sumatra and Borneo, Indonesia. PLoS One. 2016;11:e0152771.

20. Sak B, Petrzelkova KJ, Kvetonova D, Mynarova A, Shutt KA, Pomajbikova K, et al. Long-term monitoring of microsporidia, Cryptosporidium and Giardia infections in western lowland gorillas (Gorilla gorilla gorilla) at different stages of habituation in Dzanga Sangha Protected Areas, Central African Republic. PLoS One. 2013;8:e71840.

21. Sak B, Kvác M, Petrzelková K, Kvetonová D, Pomajbíková K, Mulama M, et al. Diversity of microsporidia (Fungi: Microsporidia) among captive great apes in European zoos and African sanctuaries: evidence for zoonotic transmission? Folia Parasitol (Praha). 2011;58:81 - 6.

22. Yu F, Wu Y, Li T, Cao J, Wang J, Hu S, et al. High prevalence of Enterocytozoon bieneusi zoonotic genotype $D$ in captive golden snub-nosed monkey (Rhinopithecus roxellanae) in zoos in China. BMC Vet Res. 2017;13:158.

23. Ye J, Xiao L, Li J, Huang W, Amer SE, Guo Y, et al. Occurrence of human-pathogenic Enterocytozoon bieneusi, Giardia duodenalis and Cryptosporidium genotypes in laboratory macaques in Guangxi, China. Parasitol Int. 2014;63:132-7.

24. Chen L, Zhao J, Li N, Guo Y, Feng Y, Feng Y, et al. Genotypes and public health potential of Enterocytozoon bieneusi and Giardia duodenalis in crab-eating macaques. Parasit Vectors. 2019;12:254.

25. Yu F, Li D, Chang Y, Wu Y, Guo Z, Jia L, et al. Molecular characterization of three intestinal protozoans in hospitalized children with different disease backgrounds in Zhengzhou, central China. Parasit Vectors. 2019;12:543.

26. Liu H, Jiang Z, Yuan Z, Yin J, Wang Z, Yu B, et al. Infection by and genotype characteristics of Enterocytozoon bieneusi in HIV/AIDS patients from Guangxi Zhuang autonomous region, China. BMC Infect Dis. 2017; 17:684.

27. Li N, Xiao L, Wang L, Zhao S, Zhao X, Duan L, et al. Molecular surveillance of Cryptosporidium spp., Giardia duodenalis, and Enterocytozoon bieneusi by genotyping and subtyping parasites in wastewater. PLoS Negl Trop Dis. 2012;6:e1809.

28. Matos O, Lobo ML, Xiao L. Epidemiology of Enterocytozoon bieneusi infection in humans. J Parasitol Res. 2012;2012: 981424.

29. Wang L, Zhang H, Zhao X, Zhang L, Zhang G, Guo M, et al. Zoonotic Cryptosporidium species and Enterocytozoon bieneusi genotypes in HIV-positive patients on antiretroviral therapy. J Clin Microbiol. 2013;51:557 - 63.

30. Zang M, Li J, Tang C, Ding S, Huang W, Qin Q, et al. Prevalence and phylogenetic analysis of Microsporidium Enterocytozoon bieneusi in diarrheal patients. Pathogens. 2021;10:128.

31. Qi M, Yu F, Zhao A, Zhang Y, Wei Z, Li D, Zhang L. Unusual dominant genotype NIA1 of Enterocytozoon bieneusi in children in Southern Xinjiang, China. PLoS Negl Trop Dis. 
2020; 14:e0008293.

32. Zhang W, Ren G, Zhao W, Yang Z, Shen Y, Sun Y, et al. Genotyping of Enterocytozoon bieneusi and subtyping of Blastocystis in cancer patients: relationship to diarrhea and assessment of zoonotic transmission. Front Microbiol. 2017;8:1835.

33. Wang T, Fan Y, Koehler AV, Ma G, Li T, Hu M, et al. First survey of Cryptosporidium, Giardia and Enterocytozoon in diarrhoeic children from Wuhan, China. Infect Genet Evol. 2017;51:127-131.

34. Sulaiman IM, Bern C, Gilman R, Cama V, Kawai V, Vargas D, et al. A molecular biologic study of Enterocytozoon bieneusi in HIV-infected patients in Lima, Peru. J Eukaryot Microbiol. 2003;50 Suppl:591-6.

35. Gong B, Yang Y, Liu X, Cao J, Xu M, Xu N, et al. First survey of Enterocytozoon bieneusi and dominant genotype Peru6 among ethnic minority groups in southwestern China's Yunnan Province and assessment of risk factors. PLoS Negl Trop Dis. 2019;13:e0007356.

36. Ruviniyia K, Abdullah DA, Sumita S, Lim YAL, Ooi PT, Sharma RSK. Molecular detection of porcine Enterocytozoon bieneusi infection in Peninsular Malaysia and epidemiological risk factors associated with potentially zoonotic genotypes. Parasitol Res. 2020;119:1663-1674.

37. Zhao W, Yu S, Yang Z, Zhang Y, Zhang L, Wang R, et al. Genotyping of Enterocytozoon bieneusi (Microsporidia) isolated from various birds in China. Infect Genet Evol. 2016;40:151-154.

38. Zhao W, Zhang W, Yang D, Zhang L, Wang R, Liu A. Prevalence of Enterocytozoon bieneusi and genetic diversity of ITS genotypes in sheep and goats in China. Infect Genet Evol. 2015;32:265 - 70.

39. Liu W, Nie C, Zhang L, Wang R, Liu A, Zhao W, et al. First detection and genotyping of Enterocytozoon bieneusi in reindeers (Rangifer tarandus): a zoonotic potential of ITS genotypes. Parasit Vectors. $2015 ; 8: 526$.

40. Ye J, Ji Y, Xu J, Ma K, Yang X. Zoonotic Enterocytozoon bieneusi in raw wastewater in Zhengzhou, China. Folia Parasitol (Praha). 2017;64:2017.002.

41. Dong H, Cheng R, Li X, Li J, Chen Y, Ban C, et al. Molecular Identification of Cryptosporidium spp., Enterocytozoon bieneusi, and Giardia duodenalis in captive pet birds in Henan province, central China. J Eukaryot Microbiol. 2021:e12839.

42. Li D, Zheng S, Zhou C, Karim MR, Wang L, et al. Multilocus Typing of Enterocytozoon bieneusi in pig reveals the high prevalence, zoonotic potential, host adaptation and geographical segregation in China. J Eukaryot Microbiol. 2019;66:707-718.

43. Li J, Li D, Zhang H, Wang R, Lin Z, Zhang L, et al. Molecular characterization and novel genotypes of Enterocytozoon bieneusiin pet snakes in Beijing, China. Int J Parasitol Parasites Wildl. 2020;12:172175.

44. Wang Y, Zhang K, Zhang Y, Wang K, Gazizova A, Wang L,et al. First detection of Enterocytozoon bieneusi in whooper swans (Cygnus cygnus) in China. Parasit Vectors. 2020;13:5.

45. Espern A, Morio F, Miegeville M, Illa H, Abdoulaye M, Meyssonnier V, et al. Molecular study of microsporidiosis due to Enterocytozoon bieneusi and Encephalitozoon intestinalis among human 
immunodeficiency virus-infected patients from two geographical areas: Niamey, Niger, and Hanoi, Vietnam. J Clin Microbiol. 2007;45:2999-3002.

46. Leelayoova S, Subrungruang I, Suputtamongkol Y, Worapong J, Petmitr PC, Mungthin M. Identification of genotypes of Enterocytozoon bieneusi from stool samples from human immunodeficiency virus-infected patients in Thailand. J Clin Microbiol. 2006;44:3001-4.

47. Mirjalali H, Mirhendi H, Meamar AR, Mohebali M, Askari Z, Mirsamadi ES, et al. Genotyping and molecular analysis of Enterocytozoon bieneusi isolated from immunocompromised patients in Iran. Infect Genet Evol. 2015;36:244-249.

48. Yang J, Song M, Wan Q, Li Y, Lu Y, Jiang Y, et al. Enterocytozoon bieneusi genotypes in children in northeast China and assessment of risk of zoonotic transmission. J Clin Microbiol. 2014;52:4363-7.

49. Zheng XL, Zhou HH, Ren G, Ma TM, Cao ZX, Wei LM, et al. Genotyping and zoonotic potential of Enterocytozoon bieneusi in cattle farmed in Hainan Province, the southernmost region of China. Parasite. 2020;27:65.

\section{Figures}

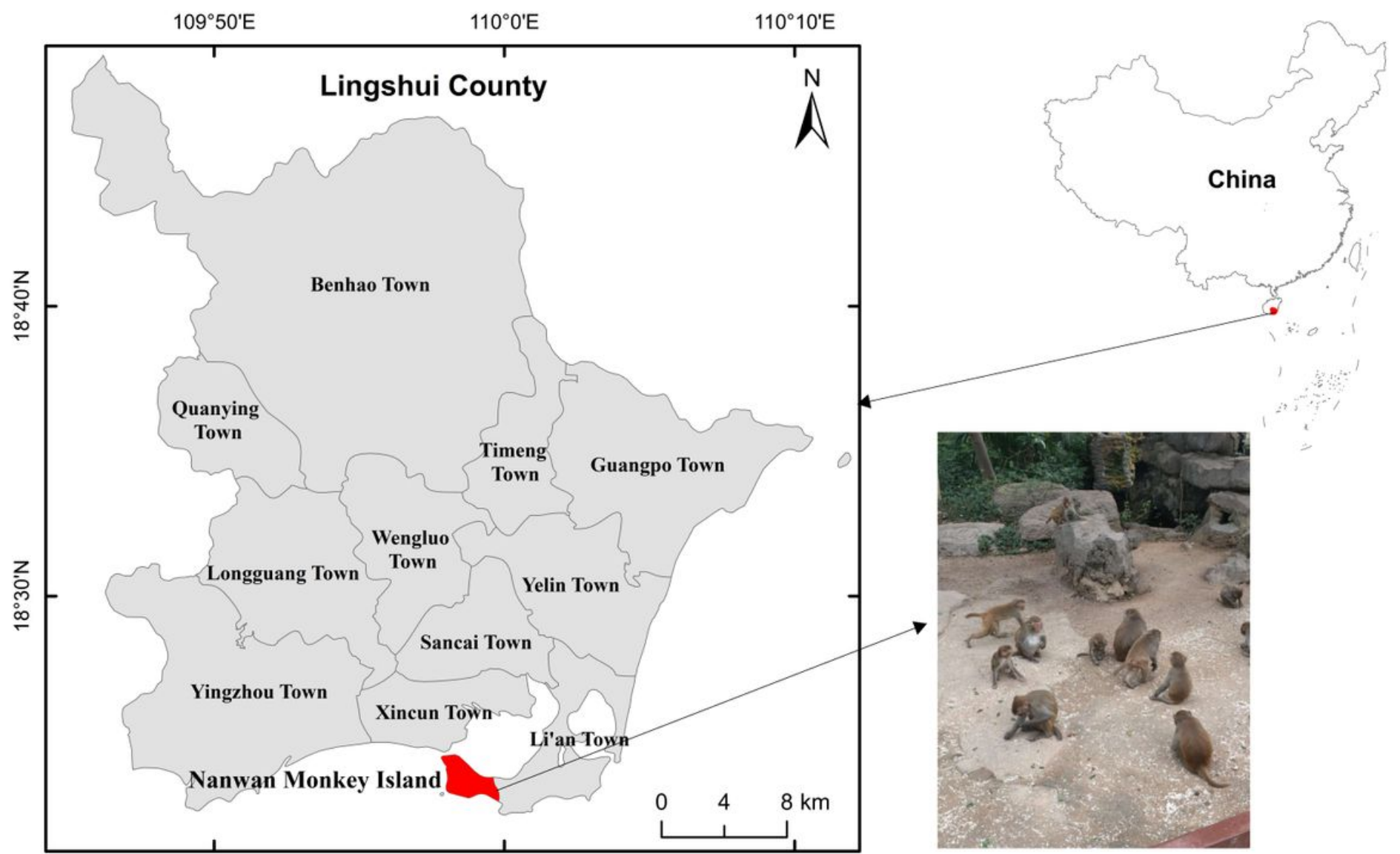

Figure 1 
The location of the Nanwan Monkey Island, Hainan of China where the location of sample collection. Note: The designations employed and the presentation of the material on this map do not imply the expression of any opinion whatsoever on the part of Research Square concerning the legal status of any country, territory, city or area or of its authorities, or concerning the delimitation of its frontiers or boundaries. This map has been provided by the authors.

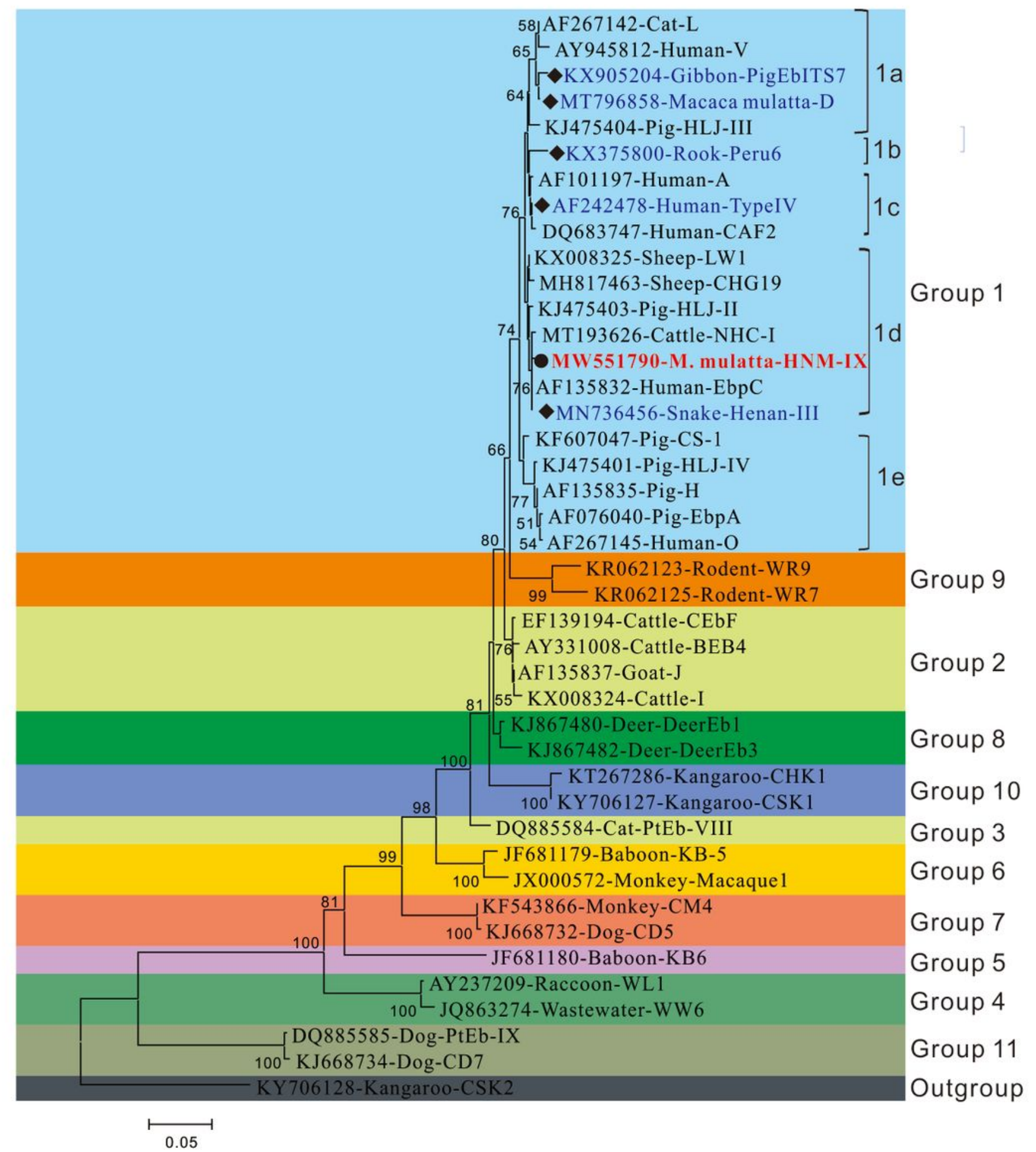

Figure 2 
Phylogenetic relationship of Enterocytozoon bieneusi genotype groups. The relationship of Enterocytozoon bieneusi genotypes identified in the present study and other known genotypes deposited in the GenBank was inferred by neighbor-joining ITS sequences analysis based on the genetic distance using the Kimura two-parameter model. The numbers on the branches are percent bootstrap values from 1,000 replicates. Each sequence is identified by its accession number, host origin, and genotype designation. The group terminology for the clusters is based on Zheng et al. [49]. The squares and circles filled in black indicate novel and known genotypes identified in this study, respectively. 